\title{
O gênero Ceratium Schrank (Dinophyta) na plataforma continental e águas oceânicas do Estado de Pernambuco, Brasil
}

\author{
Maria Luise Koening ${ }^{1,3}$ e Cynthia Gomes de Lira ${ }^{1,2}$
}

Recebido em 24/10/2002. Aceito em 26/10/2004

\begin{abstract}
RESUMO - (O gênero Ceratium Schrank (Dinophyta) na plataforma continental e águas oceânicas do Estado de Pernambuco, Brasil). Este trabalho apresenta as espécies e distribuição do gênero Ceratium no Estado de Pernambuco. As amostras foram coletadas pelo navio de pesquisas Victor Hensen durante a prospecção do JOPS II-5 no período de 25/fevereiro a 3/março/1995. Foram analisados sete perfis perpendiculares à costa, de 50 milhas de distância cada, totalizando 34 estações, distantes 10 milhas umas das outras, sendo 14 estações na região nerítica e 20 na oceânica. As amostras foram coletadas com rede tipo bongo, com abertura de malha de 64 mm e fixadas com formol neutro a 4\%. Foram identificadas 27 espécies, 24 variedades, quatro subespécies e três formas, totalizando 58 táxons. As espécies consideradas muito freqüentes foram C. contortum var. karstenii (97\%), C. macroceros var. macroceros (82\%), C. teres e C. euarcuatum (79\%), e Ceratium tripos subsp. tripos (73\%), sendo esta última a única subespécie abundante na área. Cerca de 56\% dos táxons ocorreu tanto na região nerítica como oceânica, mas a região oceânica apresentou maior riqueza de espécies por amostra analisada, sendo $42 \%$ dos táxons encontrados nesta região.
\end{abstract}

Palavras-chave: Dinoflagelados, Ceratium, distribuição, Pernambuco

\begin{abstract}
The genus Ceratium Schrank (Dinophyta) from coastal and oceanic waters of Pernambuco State, Brazil). This work presents the species and the distribution of the Ceratium Schrank (Pyrrophyta) from coastal and oceanic waters of Pernambuco State. Sampling were performed aboard the Research Vessel Victor Hensen, during the JOPS II Project, Leg 5, from February 25 to March 3 , 1995. Collections were carried out in 34 stations, distributed in 7 profiles perpendicular to the coast, with 14 stations located at the neritic region and 20 at the oceanic one. Samples were collected with a bongo net, $64 \mathrm{~mm}$ and fixed with $4 \%$ neutralized formaldehyde. Fifty eight specific and underspecific taxa were identified, outranking as the most frequent $C$. contortum var. karstenii (97\%), C. macroceros var. macroceros (82\%), C. teres, C. euarcuatum (79\%), and Ceratium tripos subsp. tripos (73\%) the last one being the only abundant subspecies. $56 \%$ of the taxa ocurred at the neritic and oceanic region. The oceanic region presented $42 \%$ of species per sample analised.
\end{abstract}

Key words: Dinoflagellates, Ceratium, distribution, Pernambuco

\section{Introdução}

Os dinoflagelados representam um dos grupos mais importantes do fitoplâncton marinho, depois das diatomáceas, e determinam direta e indiretamente a fertilidade do mar (Balech 1988).

Desempenham papel ecológico como produtores primários e como organismos heterotróficos na teia trófica do ambiente marinho. Além disso, são considerados bons indicadores biológicos das massas de água, responsáveis por grande parte da bioluminescência no mar e, algumas espécies, podem ocasionar florações nocivas, cuja amplitude e freqüência têm reflexo direto sobre a saúde humana e atividade pesqueira (Licea et al. 1995).
Algumas espécies de dinoflagelados são de águas polares, temperadas e tropicais, mas possuem tendência a serem mais comuns em águas quentes. Segundo Taylor (1987), a maioria das espécies vive em águas oceânicas com salinidade maior que 30\%, raramente maior que 40\%. Aproximadamente $90 \%$ das espécies são marinhas planctônicas ou bentônicas.

Apesar de bem representados em mares tropicais, ainda existem grandes lacunas no estudo destes organismos, talvez, pela falta de pesquisadores especializados, o que tem influído na falta de planejamento de pesquisas direcionadas ao levantamento taxonômico do grupo nos ambientes marinhos de Pernambuco. Por este motivo, no diagnóstico da biodiversidade de Pernambuco foi

\footnotetext{
1 Universidade Federal de Pernambuco, Departamento de Oceanografia, Av. Arquitetura, s/n, Cidade Universitária, CEP 50739-540, Recife, PE, Brasil

2 Bolsista PIBIC/FACEPE (Fundação de Amparo à Ciência e Tecnologia do Estado de Pernambuco)

3 Autor para correspondência: koening@ufpe.br
} 
recomendado maior incentivo no estudo desse grupo.

Grande parte das informações existentes sobre os dinoflagelados de Pernambuco está inserida em levantamentos da flora planctônica (Eskinazi-Leça \& Passavante 1972; Eskinazi-Leça et al. 1989a; b; Gomes 1990). Estudos específicos sobre o grupo, entretanto, são raros e estão limitados à região nerítica, a aproximadamente 15 milhas da costa, sendo a primeira e única contribuição a de Passavante (1979).

Durante estudo realizado em 1995, em áreas de plataforma e região oceânica adjacente à costa de Pernambuco, os indivíduos do gênero Ceratium Schrank foram os que mais se destacaram. Possuem o corpo mais ou menos achatado dorsoventralmente e caracterizam-se por possuírem cerca de dois cornos, dos quais um é apical, e o outro, ou outros dois, antiapicais. São de forma bastante característica e muito abundantes no plâncton. Nem todas as espécies são fáceis de diferenciar, pois sua distribuição muito ampla e, sobretudo, as espécies neríticas, são muito variáveis podendo ser subdivididas em categorias menores como subespécies, variedades e algumas formas (Balech 1988).

O presente trabalho tem como objetivo mapear a distribuição das espécies de Ceratium e determinar a abundância relativa e freqüência de ocorrência das espécies, ampliando desta maneira, os conhecimentos sobre os dinoflagelados no Estado de Pernambuco.

\section{Material e métodos}

O material para o presente estudo faz parte do acervo de amostras de plâncton do Departamento de Oceanografia da Universidade Federal de Pernambuco. Estas amostras foram coletadas pelo Navio de Pesquisas Victor Hensen, durante a prospecção do JOPS II-5 no período de 25/fevereiro a 3/março/1995. Foram analisados sete perfis perpendiculares à costa, de 50 milhas de distância cada, totalizando 34 estações, distantes 10 milhas umas das outras, sendo 14 neríticas e 20 oceânicas. A fronteira entre as regiões nerítica e oceânica foi definida como sendo a isóbata de $200 \mathrm{~m}$.

Os perfis estudados foram: Perfil 2-Rio Formoso; Perfil 3-Suape; Perfil 4-Rio Jaboatão; Perfil 5-Porto do Recife; Perfil 6-Itamaracá/Forte Orange; Perfil 7-Itamaracá/Catuama e Perfil 8-Rio Goiana (Fig. 1). As amostras foram coletadas por meio de uma rede tipo Baby Bongo com 2 m compr., 60 cm diâm. de boca e abertura de malha de $64 \mu \mathrm{m}$. Foram feitos arrastos oblíquos a profundidades entre $14 \mathrm{~m}$ e $0 \mathrm{~m}$

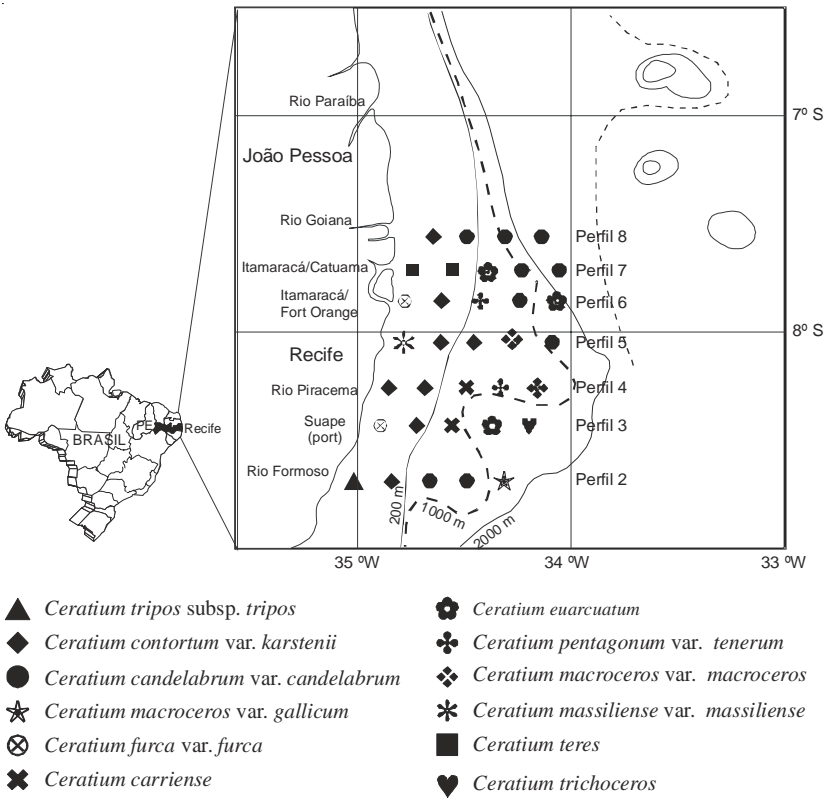

Figura 1. Espécies de maior abundância relativa nos perfis e estações do Estado de Pernambuco, Brasil.

(estações neríticas) e 160 m e 0 m (oceânicas), sendo as amostras fixadas com formol neutro a $4 \%$. Para a identificação das espécies foram consultados os trabalhos de Sournia (1967), Wood (1968), Pesantes (1978), Passavante (1979), Dodge (1982), Balech (1988), Cardoso et al. (1994), Cardoso (1995, 1997 e 1998), Licea et al. (1995) e Steidinger \& Tangen (1997).

A abundância relativa foi calculada levando-se em consideração o número total de organismos de cada táxon, em relação ao número total de organismos na amostra, tendo sido adotadas as seguintes categorias: Dominante $\geq 50 \%$; Abundante $\leq 50 \%$ - $>30 \%$; Pouco Abundante $\leq 30 \%$ - $>10 \%$ e Rara $\leq 10 \%$.

Para a freqüência de ocorrência foi levado em consideração o número de amostras nas quais cada táxon ocorreu e o número total de amostras analisadas, tendo sido adotadas as seguintes categorias: Muito Freqüente $\geq 70 \%$; Freqüente $\leq 70 \%$ - $>30 \%$; Pouco Freqüente $\leq 30 \%$ - $>10 \%$ e Esporádica $\leq 10 \%$.

\section{Resultados e discussão}

$\mathrm{Na}$ área estudada, o gênero Ceratium foi o que mais se destacou em termos de riqueza específica nas amostras analisadas, tendo sido identificadas 27 espécies, 24 variedades, quatro subespécies e três formas, totalizando 58 táxons (Tab. 1). Dentre estes táxons, 27 são novas ocorrências para Pernambuco.

As espécies mais representativas em termos de abundância relativa nos perfis estudados (Fig. 1), 
Tabela 1. Lista das espécies de Ceratium Schrank identificadas na área (segundo Licea et al. 1995 e Steidinger \& Tangen 1997) e * novas ocorrências para o Estado de Pernambuco, Brasil.

Espécies identificadas na área

Novas ocorrências

Ceratium arietinum var. arietinum Sournia

Ceratium arietinum var. gracilentum (Jörgensen) Sournia

Ceratium breve (Ostenfeld \& Schmidt) Schröder

Ceratium breve var. parallelum (Schmidt) Jörgensen

Ceratium breve var. smidtii (Jörgensen) Sournia

Ceratium candelabrum (Ehrenberg) Stein var. candelabrum

Ceratium candelabrum var. depressum (Pouchet) Jörgensen

Ceratium carriense (Gourret) var. carriense

Ceratium cephalotum (Lemmermann) Jörgensen

Ceratium concilians Jörgensen

Ceratium contortum var. contortum (Gourret) Cleve

Ceratium contortum var. karstenii (Pavillard) Sournia

Ceratium contortum var. robustum (Karsten) Sournia

Ceratium deflexum (Kofoid) Jörgensen

Ceratium digitatum Schütt

Ceratium euarcuatum Jörgensen

Ceratium extensum (Gourett) Cleve

Ceratium falcatiforme Jörgensen

Ceratium furca Claparède \& Lachmann var. furca

Ceratium fusus (Ehrenberg) Dujardin var. fusus

Ceratium fusus var. seta (Ehrenberg) Sournia

Ceratium geniculatum (Lemmermann) Cleve

Ceratium gibberum Gourret

Ceratium hexacanthum Gourett

Ceratium horridum var. horridum (Sournia)

Ceratium horridum var. buceros (Zacharias Sournia

Ceratium incisum (Karsten) Jörgensen

Ceratium kofoidii Jörgensen

Ceratium limulus Gourret

Ceratium lineatum (Ehrenberg) Cleve

Ceratium longirostrum Gourret

Ceratium lunula (Schimper ex Karsten) Jörgensen

*Ceratium arietinum var. arietinum

*Ceratium arietinum var. gracilentum

*Ceratium breve var. parallelum

*Ceratium breve var. smidtii

*Ceratium concilians Jörgensen

*Ceratium contortum var. karstenii

* Ceratium deflexum

* Ceratium digitatum

* Ceratium euarcuatum

*Ceratium falcatiforme

* Ceratium fusus var. seta

*Ceratium horridum var. buceros

* Ceratium incisum

* Ceratium lunula

*Ceratium paradoxides

* Ceratium pentagonum var. subrobustum

* Ceratium pentagonum var. tenerum

* Ceratium platycorne

* Ceratium praelongum

* Ceratium ranipes

* Ceratium symmetricum var. coarctatum

* Ceratium symmetricum var. symmetricum

* Ceratium tenue subsp. tenue

* Ceratium tenue subsp. tenuissimum

* Ceratium tripos subsp. tripodioides

* Ceratium vultur f. summatranum

* Ceratium vultur f. recurvum

Ceratium macroceros (Ehrenberg) Vanhöffen var. macroceros

Ceratium macroceros var. gallicum (Kofoid) Sournia

Ceratium massiliense (Gourret) Karsten var. massiliense

Ceratium massiliense f. armatum (Karsten) Jörgensen

Ceratium paradoxides Cleve

Ceratium pavillardii Jörgensen

Ceratim pentagonum Gourret

Ceratium pentagonum var. subrobustum Jörgensen

Ceratium pentagonum var. tenerum Jörgensen

Ceratium platycorne Daday

Ceratium praelongum (Lemmermann) Kofoid ex Jörgensen

Ceratium ranipes Cleve

Ceratium reflexum Cleve

Ceratium symmetricum var. coarctatum (Pavillard) Graham \& Bronikowsky

Ceratium symmetricum var. symmetricum Graham \& Bronikowsky

Ceratium tenue subsp. tenue (Ostenfeld) \& Schmidt

Ceratium tenue subsp. tenuissimum (Kofoid) Graham \& Bronikovsky

Ceratium teres Kofoid

Ceratium trichoceros (Ehrenberg) Kofoid

Ceratium tripos var. pulchellum (Schröder) López ex Sournia

Ceratium tripos tripos Balech

Ceratium tripos tripodioides Jörgensen

Ceratium vultur Cleve

Ceratium vultur var. japonicum (B. Schröder) (Jörgensen)

Ceratium vultur f. summatranum (Karsten) Sournia

Ceratium vultur f. recurvum (Jörgensen) Schiller 
apresentaram-se distribuídas da seguinte maneira:

$\mathrm{Na}$ região nerítica, ocorreram Ceratium contortum var. karstenii, C. teres, C. furca var. furca, C. massiliense var. massiliense e C. tripos subsp. tripos, destacando-se Ceratium contortum var. karstenii nestas estações. Na região oceânica, ocorreram Ceratium candelabrum var. candelabrum, Ceratium euarcuatum, C. carriense, C. macroceros var. macroceros, C. macroceros var. gallicum, $C$. pentagonum var. tenerum e C. trichoceros, predominando Ceratium candelabrum var. candelabrum. Em ambas as regiões ocorreram Ceratium contortum var. karstenii e Ceratium candelabrum var. candelabrum.

As Fig. 2-21 apresentam algumas das espécies ocorrentes na área de estudo.

Algumas espécies identificadas destacaram-se pela sua freqüência. Dentre as espécies muito freqüentes destacaram-se: C. contortum var. karstenii (97\%), C. macroceros var. macroceros (82\%), C. teres e C. euarcuatum (79\%), e Ceratium tripos subsp. tripos (73\%), sendo esta última, a única subespécie abundante na área, com 33\% de abundância relativa, na estação 7 (Rio Formoso). A maioria das espécies foi pouco abundante e rara nas estações estudadas.

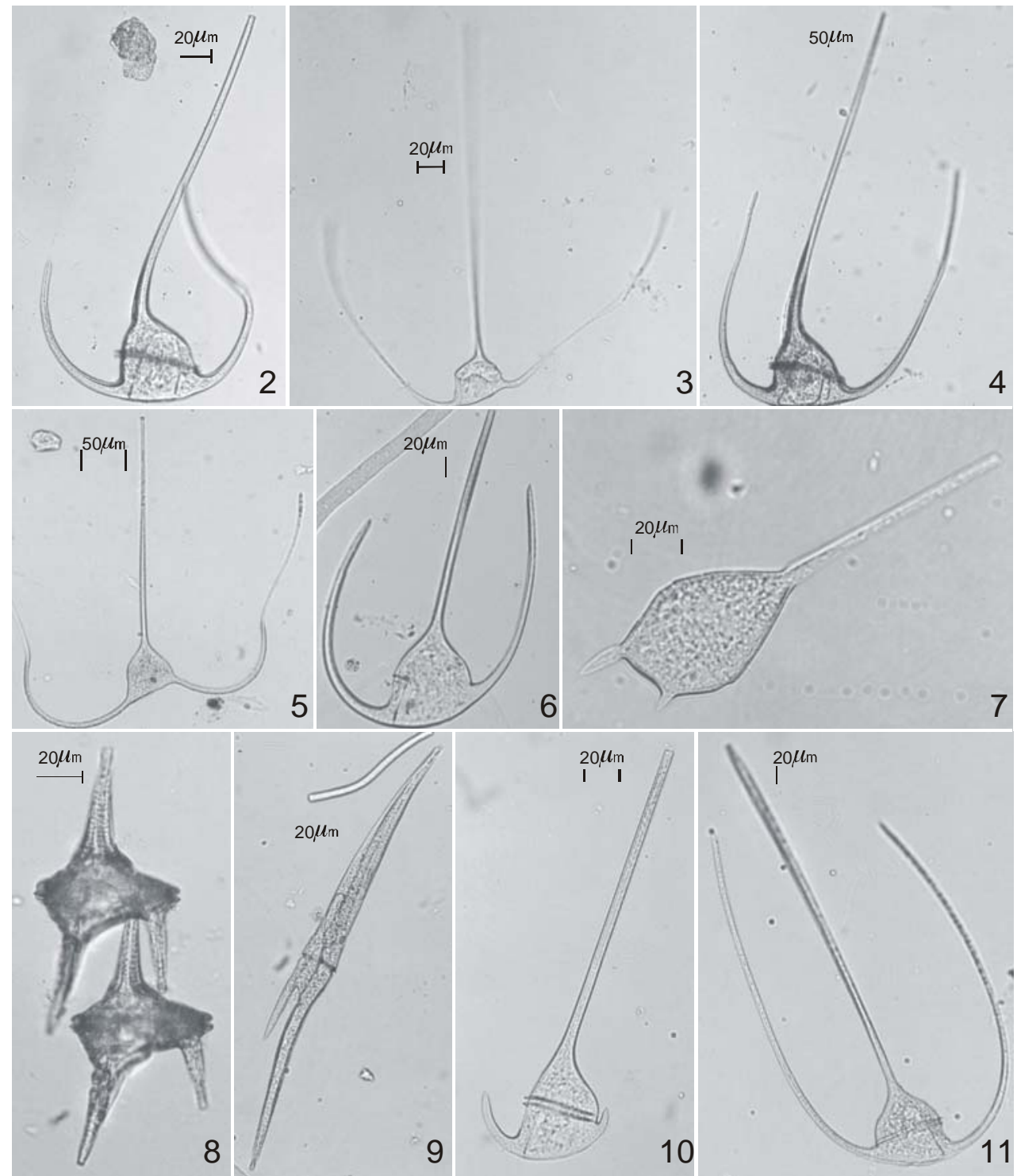

Figuras 2-11. 2. Ceratium contortum var. contortum (Gourret) Cleve. 3. Ceratium carriense (Gourret) var. carriense. 4. Ceratium contortum var. karstenii (Pavillard) Sournia. 5. Ceratium trichoceros (Ehrenberg) Kofoid. 6. Ceratium euarcuatum Jörgensen. 7. Ceratium teres Kofoid. 8. Ceratium candelabrum (Ehrenberg) Stein var. candelabrum. 9. Ceratium incisum (Karsten) Jörgensen. 10. Ceratium tripos var. pulchellum (Schröder) López ex Sournia. 11. Ceratium symmetricum var. coarctatum (Pavillard) Graham \& Bronikowsky. 
O número de espécies de Ceratium por estação variou de um mínimo de duas espécies no estuário do rio Formoso (Est. 7 - Perfil 2) a um máximo de 43 espécies no estuário do rio Jaboatão (Est. 22 - Perfil 4). As estações costeiras apresentaram menor riqueza de espécies em relação às estações oceânicas.

No presente trabalho, aproximadamente $42 \%$ das espécies ocorreram apenas em águas oceânicas, 2\% em águas neríticas e 56\% ocorreram em ambas.

Os trabalhos sobre os dinoflagelados encontrados em águas marinhas da costa do Brasil como os de Brandini (1988) Brandini \& Morais (1986) MacêdoSaidah \& Moreira Filho (1977) referem-se à esses organismos em estudos sobre composição e distribuição do fitoplâncton. Trabalhos específicos sobre o grupo são os de Kremer \& Rosa (1983) Cardoso et al. (1994) Cardoso (1995; 1997; 1998), entretanto, esses trabalhos encontram-se concentrados no sudeste do Brasil.

Dos 58 táxons identificados no presente trabalho, 20 táxons (Ceratium breve, C. candelabrum var. candelabrum, C. candelabrum var. depressum, C. carriense, C. contortum var. karstenii, C. furca var. furca, C. fusus var. fusus, C. fusus var. seta, C. gibberum, C. hexacanthum, C. lineatum, C. massiliense var. massiliense, $C$. pentagonum var.

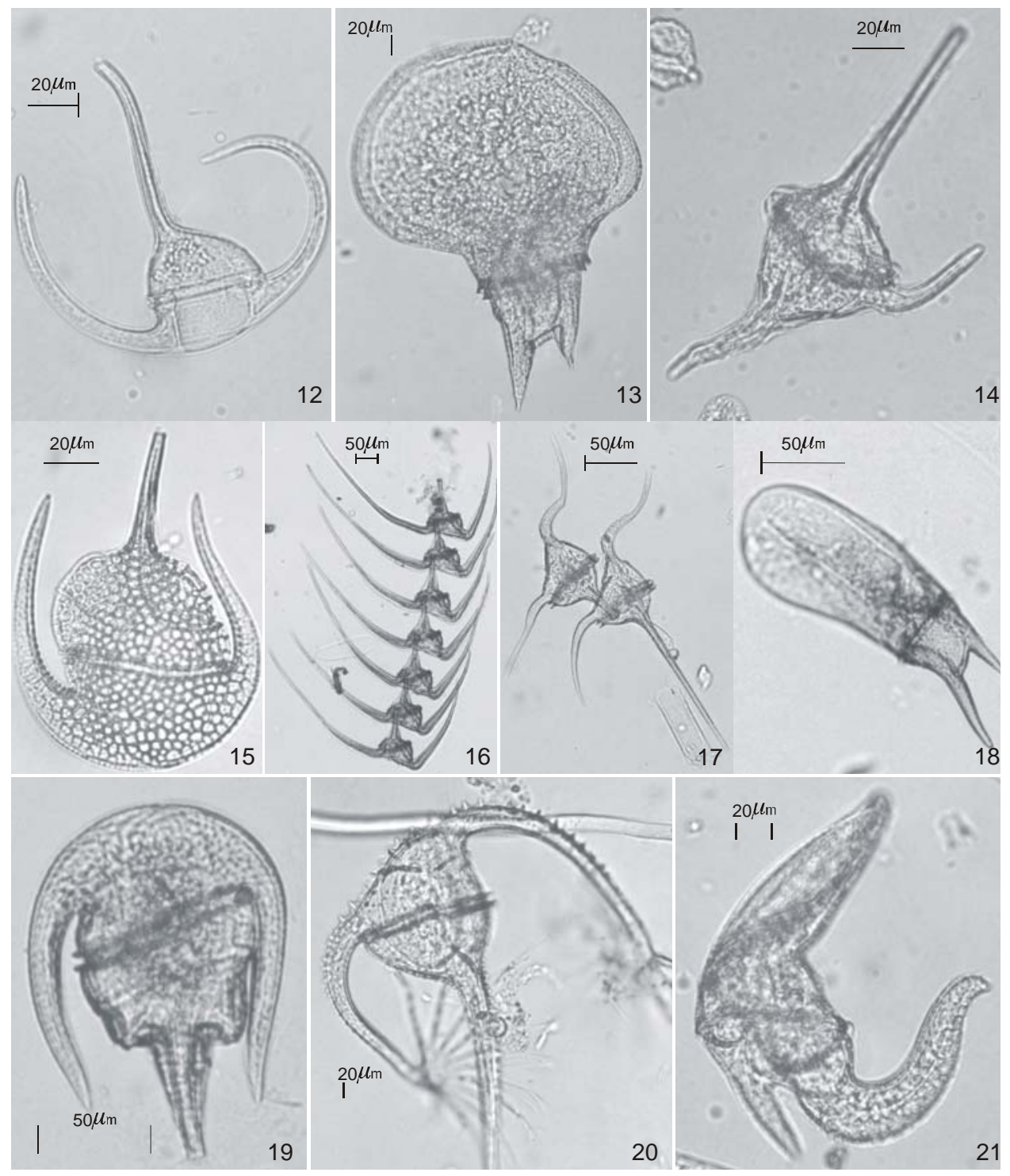

Figuras 12-21. 12. Ceratium arietinum var. arietinum Sournia. 13. Ceratium cephalotum (Lemmermann) Jörgensen. 14. Ceratium reflexum Cleve. 15 Ceratium paradoxides Cleve. 16. Ceratium vultur f. summatranum (Karsten) Sournia (em cadeia). 17 Ceratium vultur f. recurvum (Jörgensen) Schiller. 18. Ceratium praelongum (Lemmermann) Kofoid ex Jörgensen. 19. Ceratium limulus Gourret. 20. Ceratium ranipes Cleve. 21. Ceratium digitatum Schütt. 
subrobustum, C. pentagonum var. tenerum, C. ranipes, C. teres, C. trichoceros, C. tripos var. pulchellum, C. tripos subsp. tripos e C. vultur) foram comuns tanto na região tropical (NE) como na subtropical (S) do Brasil.

Kremer \& Rosa (1983) identificaram 18 espécies de Ceratium para Tramandaí (Rio Grande do Sul) e Cardoso (1995), estudando o gênero Ceratium da Ilha do Arvoredo e Praia de Ponta das Canas (Santa Catarina) identificou 21 táxons específicos e infraespecíficos. Os autores mencionaram a preferência das espécies desse gênero por águas tropicais e subtropicais, ocorrendo tanto na região nerítica como oceânica.

Na Plataforma Continental de Pernambuco, a maioria dos trabalhos estão limitados à região nerítica, à aproximadamente 15 milhas da costa (Eskinazi-Leça \& Passavante 1972; Eskinazi-Leça et al. 1989a; b; Gomes 1990) e são listagens taxonômicas sobre o fitoplâncton, o que não permite uma discussão sobre o grupo.

A primeira contribuição detalhada dos dinoflagelados com dados ecológicos e de distribuição foi a de Passavante (1979) que registrou 43 táxons específicos e infraespecíficos, sendo 16 citados pela primeira vez para o Brasil e 30 pertencentes ao gênero Ceratium. O autor destacou que a maioria das espécies é cosmopolita, ocorrendo tanto na região nerítica como oceânica.

Koening \& Macêdo (1999) identificaram nas águas costeiras e oceânicas do litoral Norte de Pernambuco (Perfil 7 - Itamaracá) 49 espécies de dinoflagelados, sendo 19 do gênero Ceratium. Essas espécies foram mais abundantes nas estações oceânicas.

Os dados obtidos concordam com a distribuição e limites de salinidade e temperatura registrados pelos autores acima e por Taylor (1987). Segundo Medeiros et al. (1999), nos perfis estudados os teores de salinidade oscilaram de $35 \%$ a $37 \%$ e a temperatura de $20^{\circ} \mathrm{C}$ a $29^{\circ} \mathrm{C}$ sendo a massa de água presente na área caracterizada como Água Tropical (AT).

Pode-se verificar que o conjunto desses fatores, ou seja, o regime hidrológico na área, certamente contribuiu para a alta riqueza de espécies com valores mais baixos nas estações mais costeiras e mais altos em direção às estações oceânicas. Margalef (1978) atribuiu ainda esta maior riqueza de espécies, a maior adaptação deste grupo a ambientes de maior estabilidade ambiental e baixas concentrações de nutrientes; e esta estratégia de vida, representa um estágio de sucessão mais avançado correlacionado com um certo grau de heterotrofia e o desenvolvimento de mecanismos contra o "grazing” e predadores.

\section{Referências bibliográficas}

Balech, E. 1988. Los Dinoflagelados del Atlantico Sudoccidental. Madrid, Instituto Español de Oceanografia (Publicaciones especiales, ํㅜ 1) 310 p.

Brandini, F.P. 1988. Composição e distribuição do fitoplâncton na região sudeste do Brasil e suas relações com as massas de água (Operação Sudeste-Julho/ Agosto 1982). Ciência e Cultura 40(4): 334-341.

Brandini, F.P. \& Morais, C.L.B. 1986. Composição e distribuição do fitoplâncton em áreas costeiras e oceânicas da região sudeste do Brasil. Nerítica 1(3): 9-19.

Cardoso, L.S. 1995. O gênero Ceratium Schrank (Dinophyta, Ceratiaceae) na Ilha do Arvoredo e na Praia de Ponta das Canas, Santa Catarina, Brasil. Biociências 3(1): 3-41.

Cardoso, L.S. 1997. O gênero Protoperidinium Bergh (Dinophyta, Peridiniaceae) na Ilha do Arvoredo e na Praia de Ponta das Canas, Santa Catarina, Brasil. Biociências 5(1): 3-33.

Cardoso, L.S. 1998. Dinoflagelados da Ilha do Arvoredo e da Praia de Ponta das Canas, Santa Catarina, Brasil. Biociências 6(1): 3-54.

Cardoso, L.S.; Baptista, L.R.M. \& Jebram, D.H.A. 1994. Dinoflagelados da Ilha do Arvoredo e da Praia de Ponta das Canas, Santa Catarina, Brasil. Iheringia, série Botânica 45: 143-155.

Dodge, J.D. 1982. Marine dinoflagellates of the British Isles. London, Hobbs the Printers of Southhampton.

Eskinazi-Leça, E. \& Passavante, J.Z.O. 1972. Estudo da Plataforma Continental na área do Recife (Brasil). IV. Aspectos quantitativos do fitoplâncton. Trabalhos Oceanográficos da Universidade Federal de Pernambuco 13: 83-106.

Eskinazi-Leça, E.; Koening, M.L.; Silva, M.G.G. \& Sant’Anna, E.E. 1989a. Hidrologia e plâncton da Plataforma Continental de Pernambuco. 3. Fitoplâncton. Pp. 373-402. In: III Encontro Brasileiro de Gerenciamento Costeiro. Fortaleza.

Eskinazi-Leça, E.; Silva-Cunha, M.G.G. \& Koening, M.L. 1989b. Variação quantitativa do fitoplâncton na Plataforma Continental de Pernambuco (Brasil). Insula 19: 37-46.

Gomes, N.A. 1990. Estrutura e composição florística do fitoplâncton na plataforma continental norte de Pernambuco (Brasil). Pp. 35-53. In: Anais IV Encontro Brasileiro de Plâncton. Recife.

Koening, M.L. \& Macêdo, S.J. 1999. Hydrology and phytoplankton community structure at ItamaracáPernambuco (Northeast Brazil). Brazilian Archives of Biology and Technology 42(2): 381-392.

Kremer, L.M. \& Rosa, Z.M. 1983. Dinoflagelados do microfitoplâncton de Tramandaí. Iheringia, série Botânica 30: 3-35.

Licea, L.; Moreno, J.L.; Santoyo, H. \& Figueroa, G. 1995. Dinoflagelados del Golfo de California. Universidad Autonoma de Baja California Sur. 
Macedo-Saidah, F.E. \& Moreira Filho, H. 1977. Composição e distribuição do microfitoplâncton nas águas do Atlântico Leste do Brasil. Publicação do Instituto de Pesquisas da Marinha 107: 1-12.

Margalef, R. 1978. Life-forms of phytoplankton as survival alternatives in an unstable environment. Oceanologica Acta 1(4): 493-509.

Medeiros, C.; Macedo, S.J.; Feitosa, F.A.N. \& Koening, M.L. 1999. Hydrography and phytoplankton biomass and abundance of North-East Brazilian waters. Archive of Fishery and Marine Reseach 47(2/3): 133-151.

Passavante, J.Z.O. 1979. Contribuição ao estudo dos dinoflagelados da Plataforma Continental de Pernambuco-Brasil. Trabalhos Oceanográficos da Universidade Federal de Pernambuco 14: 31-54.
Pesantes, F. 1978. Dinoflagelados del Fitoplancton del Golfo de Guayaquil. Guayaquil, Instituto Oceanográfico Armada del Ecuador.

Sournia, A. 1967. Le genre Ceratium (Peridinien Planctonique) dans le Canal Mozambique : contribuition a une révision mondiale. Vie et Milieu, Serie A, Biologie Marine 2(3): 375-499.

Taylor, F.J.R. 1987. Dinoflagellates Morphology. In: F.J.R. Taylor (ed.). The Biology of Dinoflagellates. London, Balckwell Scientific Publications. Botanical Monographs.

Steidinger, K.A. \& Tangen, K. 1997. Dinoflagellates. Pp. 387-589. In: C.R. Tomas. Identifying Marine Phytoplankton. San Diego, Academic Press.

Wood, E.J.F. 1968. Dinoflagellates of the Caribbean Sea and Adjacents Areas. Miami, University of Miami Press. 